\title{
Registration of acoustic emission in composite material by fiber-optic sensors based on adaptive interferometer
}

\author{
Bashkov O.V., Zaikov V.I., Khon H., Bryansky A.A., \\ Bashkov I.O. \\ Materials Science Department \\ Komsomolsk-on-Amur State Technical University \\ Komsomolsk-on-Amur, Russia \\ bashkov_ov@mail.ru
}

\author{
Romashko R.V. \\ Institute of Automation and Control Processes \\ Photonics and Optical Nanometry Laboratory \\ Vladivostok, Russia \\ romashko@iacp.dvo.ru
}

\begin{abstract}
Paper presents the results of studying the features of recording acoustic emission waves recorded by fiber-optic sensors. Fiber-optic sensors are used as an elastic medium that receives acoustic emission waves. For this purpose, the optical fibers are embedded in the polymer composite material during the manufacturing of the sample. The measuring system is constructed on the basis of an adaptive interferometer. Optical fibers are part of the interferometer optical scheme. The elastic wave acting on an optical fiber changes its optical density, changing the interference pattern of the optical beam. The work of the interferometer is based on the adaptive change in the hologram, formed in the photorefractive crystal during the elastic action on the fiber. Sensitivity of fiber-optic sensors based on an adaptive interferometer allows recording acoustic emission waves. The Su-Nielsen source was used to generate the waves. The nature of the sensors, distributed in space and the anisotropy of the properties of the composite material, influenced the difference in the speed of sound in different directions and the dispersion of the velocity of the group Lamb wave. To form the signal front, the wavelet transformation was used.
\end{abstract}

Keywords-acoustic emission; adaptive interferometer; fiberoptical sensor, wavelet; sound velocity; structural health monitoring; polymer composite material

\section{INTRODUCTION}

In recent years, systems of structural health monitoring have been introduced in aviation and other modes of transport and hazardous production facilities [1-4]. One of the informative methods for monitoring the state and damage is the method of acoustic emission (AE). Piezoelectric transducers are mainly used in this method. The advantage of AE sensors is their high sensitivity, a disadvantage - low noise immunity and high cost. $\mathrm{AE}$ is also used for monitoring longterm critical objects. PZT have high sensitivity. However, large-sized objects require long cables. This reduces the noise immunity of the recording channel. The use of wireless sensors is also associated with various kinds of restrictions related to the reliability of the information channel. Fiber-optic sensors have recently been used not only as a high-speed noise-free channel for transmitting analog and digital information, but also as sensors. Fiber-optic sensors have a high linearity of the amplitude-frequency response and electromagnetic noise protection. Fiber-optic sensors on Bragg diffraction gratings are widely used $[5,6]$. In view of the high sensitivity of the sensors, their main application is the measurement of stresses and deformations similar to localized strain gauges or piezoelectric sensors.

The optical fiber laid in the inspection object can be a sensor itself due to a change in the optical density during the passage of deformation waves. The use of fiber-optical sensors (FOS) as distributed transducers of acoustic oscillations will allow expanding the scope of their application when monitoring the technical condition of extended objects.

Various schemes of constructing interferometers allow increasing the sensitivity of sensors. This became possible with the use of adaptive laser interferometers. The functioning of sensors is based on the interaction of laser radiation beams on a dynamic hologram, which is formed in a photorefractive crystal. The high sensitivity of the measuring system is achieved when two basic approaches are realized: ensuring the exact matching of the wavefronts of the reference and object light beams and maintaining the necessary phase difference between the interfering waves [6].

The paper presents the results of investigation of $\mathrm{AE}$ waves recorded by fiber-optic sensors embedded in a plate of fibrous polymer composite material (PCM). Optical fibers were built into the PCM during the laying of the fiberglass.

\section{MATERIALS AND METHODS OF RESEARCH}

A sample of the PCM plate was made by a vacuumautoclave method. Optical fibers with a diameter of $0.125 \mathrm{~mm}$ were located between the layers of the fiberglass during the manufacture of the PCM plate. Optical fibers were located along and across the plate. The plate size was $290 \mathrm{~mm} \times 130 \mathrm{~mm}$. Optical fibers were included in the optical scheme of the interferometer. The two-beam Mach-Zehnder interferometer, which is a modification of the Jamena interferometer, has a fairly high sensitivity when using an optical fiber as a sensor and an information channel. Due to the high sensitivity, the reference channel of the fiber optic sensor is exposed to uncontrolled changes in external conditions, which leads to the need for a constant restoration of the original phase difference. To solve the problem of the fading the operating point of the Mach-Zehnder 
interferometer, various methods for demodulating the output signal of a fiber-optic sensor are used. However, homodyne detection methods have some drawbacks. One of the effective solutions to the stabilization of the operating point of the interferometer is the use of dynamic holograms, which make the interferometer adaptive. An effective system for measuring small phase changes in conditions of uncontrolled environmental influences is adaptive interferometers based on the use of two-wave mixing in photorefractive crystals (PRC) [6-8]. To reduce the fading of the operating point, an adaptive interferometer was used, realized by the scheme of two-beam interaction of laser radiation on a dynamic hologram in PRC. The use of PRC positively affects the noise immunity and sensitivity of the adaptive interferometer in comparison with the use of the traditional Mach-Zehnder interferometer.

Thus, the possibility of detection of acoustic waves by fiber-optic sensors built into the PCM plate was investigated. In the detection system were used four optical fibers arranged in pairs perpendicular to each other (two on the base and two on the weft), which were included in the optical scheme of the adaptive interferometer (Fig. 1).

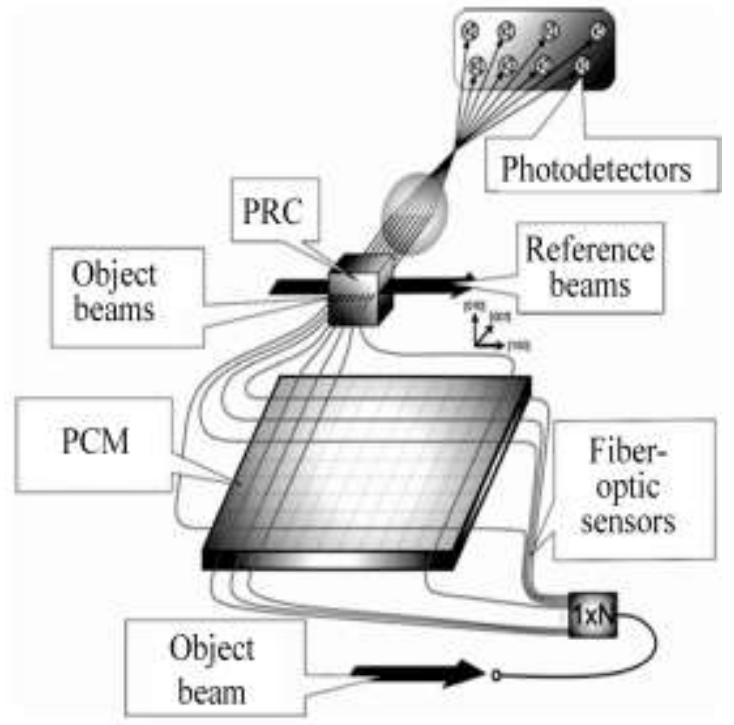

Fig. 1. Optoelectronic circuit of a multichannel fiber-optic sensor

The interferometer is realized according to the scheme of two-beam interaction of the laser radiation on a dynamic hologram in a photorefractive crystal. The use of the photorefractive crystal allows to reduce the fading of the operating point, which positively affects the noise immunity and sensitivity of the adaptive interferometer in comparison with the use of the traditional Mach-Zehnder interferometer. As a photorefractive crystal, a cadmium telluride crystal CdTe was used in these studies. The characteristic time for rerecording the hologram in a CdTe crystal is less than $1 \mathrm{~s}$. In this case, the correlation filter will adapt not only to the temperature fading of the operating point, but also to the useful signal, which can have frequencies of more than $1 \mathrm{~Hz}$.

The radiation generated by the dynamic hologram was recorded by four photodetectors connected to the recording high-frequency oscillograph. Interference of the object beams of the laser radiation passing through the optical fibers rigidly embedded in the PCM sample was performed with a single reference of the wave beam. Simultaneous recording of signals was carried out by photodetectors during the multiplexing of the dynamic holograms in a crystal [9].

To compare the signals of $\mathrm{AE}$, recorded by fiber optic sensors, with traditionally used piezoelectric transducers, specialized narrowband acoustic emission converters GT200 with an operating frequency of $165 \mathrm{kHz}$ were additionally used. Four GT200 PZT were installed at the corners of the sample (Fig. 2). A 4-channel AE-system based on the highfrequency analog-digital converter PCI-9812 was used for this [10].

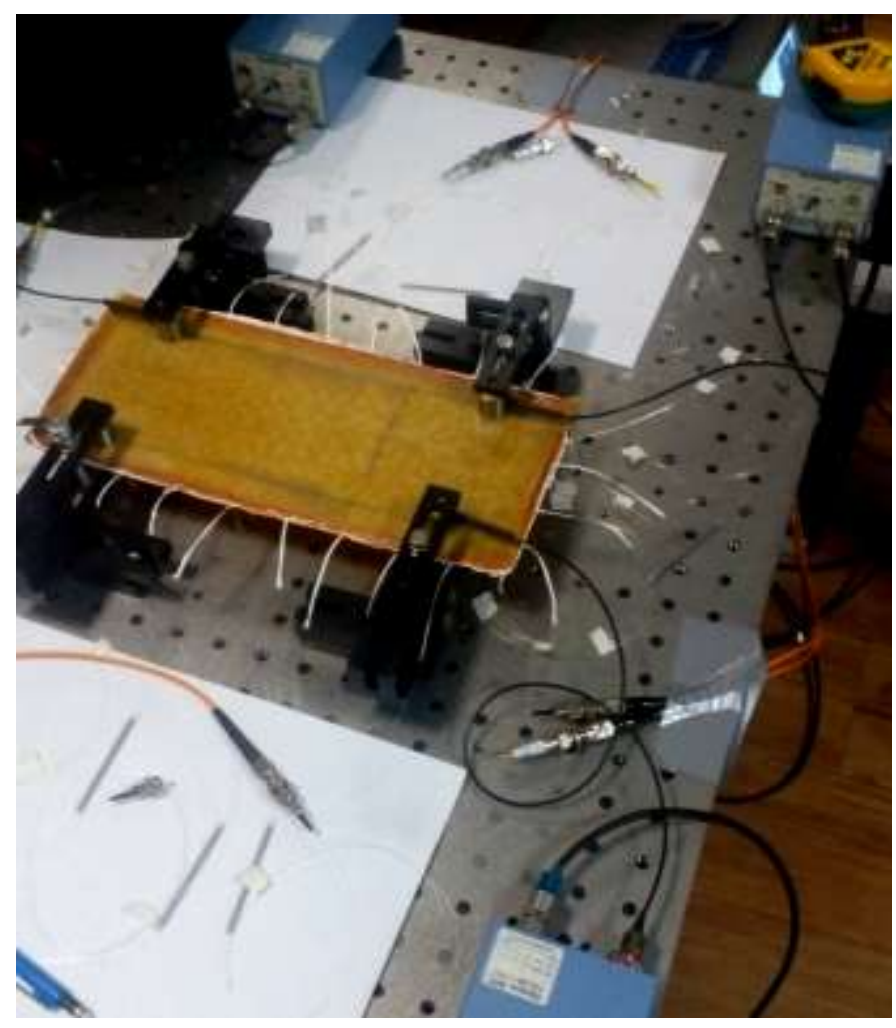

Fig. 2. PCM plate with installed PZT and FOS

The converters were connected to the $\mathrm{AE}$ system through the preamps with a gain of $40 \mathrm{~dB}$. The simulation of $\mathrm{AE}$ waves was performed by the Hsu-Nielsen source (a fracture of $2 \mathrm{H}$ pencil lead with a diameter of $0.5 \mathrm{~mm}$ ) at different locations of the plate. The purpose of this was to establish the comparative sensitivity of FOS and PET and to analyze the nature of the propagation of the acoustic waves in an anisotropic polymer composite [11].

The geometric location of FOS and PZT sensors on the PCM plate is shown in Fig. 3.

The AE source was located at a different distance from all four FOS and PZT. The direction along the long side of the PCM plate corresponds to a weft of fiberglass cloth. The direction along the short side of the PCM plate corresponds to warp of fiberglass cloth. Strength along the long side of the plate is lower than that along the short side. 


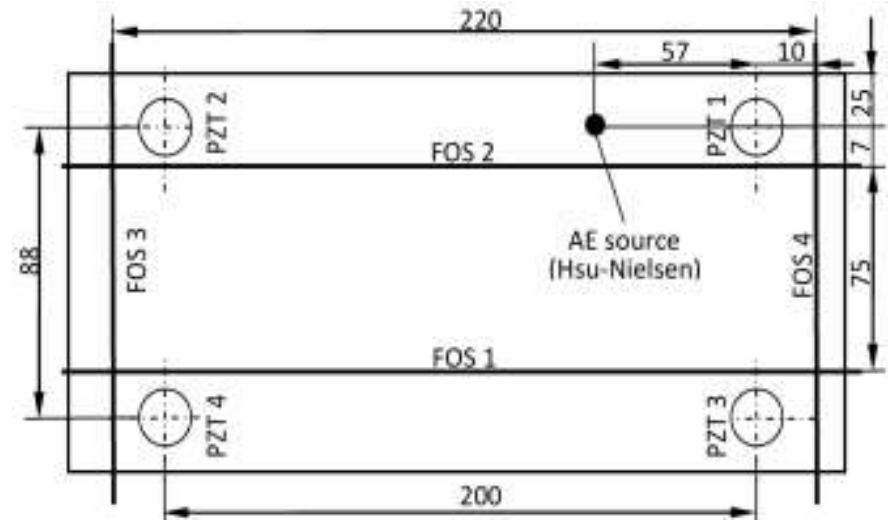

Fig. 3. Location of the PZT and FOS on the PCM plate

The anisotropy of the mechanical properties was confirmed experimentally in the bending and tensile testing of the PCM samples along a weft and warp of fiberglass cloth. The same changes correspond to the sound velocity along mutually perpendicular directions.

However, the numerical values of the properties in the diagonal direction are not intermediate between the values along and across the plate. This is due to the peculiarity of the structure of the composite. To confirm this, the sound velocity was measured in different directions: along the long side of the plate, along the short side of the plate and diagonally in the direction of the location of the PZT 1-4 and the PZT 2-3. When using FOS, it is possible to determine the sound velocity only in directions perpendicular to the direction of the location of the optical fibers. This is due to the fact that the sound wave from the point source in the plate propagates uniformly in the radial directions. The shortest distance is a perpendicular dropped to the line along which the optical fiber passes. The sound velocity along the diagonal of the PCM plate can be measured, if the optical fiber is located perpendicular to the direction of the wave propagation.

\section{RESULTS AND DISCUSSION}

The signals generated in the plate are located in the far field for most of the sensors. This affects the accuracy of changing a recording time of signals, which traces the location of the AE sources.

The technology of the PCM manufacturing with a certain direction of the fiberglass laying provides the anisotropy of the composite material's properties [12]. This is necessary to ensure the high strength in the certain direction in the real products from PCM. However, the anisotropy of properties also affects the anisotropy of the sound speed in the different directions. The signals of the $\mathrm{AE}$ were excited by a pencil break in the hardness of $2 \mathrm{H}$ (Hsu-Nielsen source) in various places of the plate and were analyzed. The paper presents the results of the investigation of signals obtained with the use of PZT and FOS when the AE wave is excited by the AE source (Hsu-Nielsen) as shown in Fig. 3.

Uneven mutual arrangement of the AE source and PZT sensors makes it possible to estimate an effect of material's anisotropy on the multidirectional sound velocity. The coordinates of the sensors and the recording time of the signals by different sensors are shown in table. 1 . The position of PZT sensor 4 is taken as the "0" coordinate.

\section{TABLE I. COORDINATES OF THE PZT AND TIME DELAY OF THE WAVE}

\begin{tabular}{|l|c|c|c|c|}
\hline & PZT 1 & PZT 2 & PZT 3 & PZT 4 \\
\hline $\mathbf{X}, \mathbf{m m}$ & 200 & 0 & 200 & 0 \\
\hline Y, mm & 88 & 88 & 0 & 0 \\
\hline Time, $\boldsymbol{\mu s}$ & 205.6 & 231.0 & 215.8 & 244.6 \\
\hline
\end{tabular}

The signals recorded by PZT are shown in Fig. 4. AE signals are group waves consisting of a longitudinal and transverse wave and a Lamb wave. Different types of waves differ in amplitude, which affects the shape of the group wave. AE source was located on the axis of PZT 1 and PZT 2 sensors at a distance of $57 \mathrm{~mm}$ from the PZT 1 sensor. Since the first wave reached sensor PZT 1, it would be reliable to calculate the propagation velocity of the wave signal in the direction of PZT 2, PZT 3, PZT 4 sensors.

The multidirectional speeds were determined by standard methods for the delay time of the wave propagation relative to the time of signal recorded by the PZT 1 sensor. The velocity of the AE wave propagation from the source to sensors PZT 2, PZT 3, PZT 4 is: $\mathrm{c} 2=3858 \mathrm{~m} / \mathrm{s}, \mathrm{c} 3=3918 \mathrm{~m} / \mathrm{s}, \quad \mathrm{c} 4=3050$ $\mathrm{m} / \mathrm{s}$, as a result of calculation. Such significant difference of the propagation speeds of the wave is due to the peculiarity of the composite structure. The structure and density of fibers in different directions (on the warp, on the weft) differ. If the direction of wave propagation coincides with one of the directions of arrangement of the fibers, the bulk of the elastic wave's energy propagates along the glass fibers that form the strands. The sound velocity in different glasses can be from 4400 to $5800 \mathrm{~m} / \mathrm{s}$. The strands of fibers are not straight, which leads to a decreasing of the sound velocity in the direction of the locating strands. The sound velocity in the cured epoxy varies from $2400 \mathrm{~m} / \mathrm{s}$ to $3000 \mathrm{~m} / \mathrm{s}$. The porosity has a significant effect on the sound velocity in the epoxy and the composite. The presence of the pores and structural nonequilibrium affects not only the attenuation of the signal.

Simultaneously with the recording of the signals by PZT sensors, registration by FOS was conducted too. The location of the optical fibers relative to the AE source is not localized. FOS are distributed sensors. This must be taken into account when choosing a method of calculating the location of $\mathrm{AE}$ sources. FOS are the broadband sensors and have a more linear frequency response than PZT sensors do. Therefore, basically, it is necessary to take into account only the geometric dimensions and properties of the material in which $\mathrm{AE}$ signals are detected, in calculations related to the propagation of waves.

Fig. 5 shows the waveform of the signals registered by FOS. Despite the low FOS sensitivity, when adjusting the optical scheme, it was possible to detect the signals against the background of noise. The shape of the signals with a long after-sound is a consequence of the resonant properties of the PCM plate. 

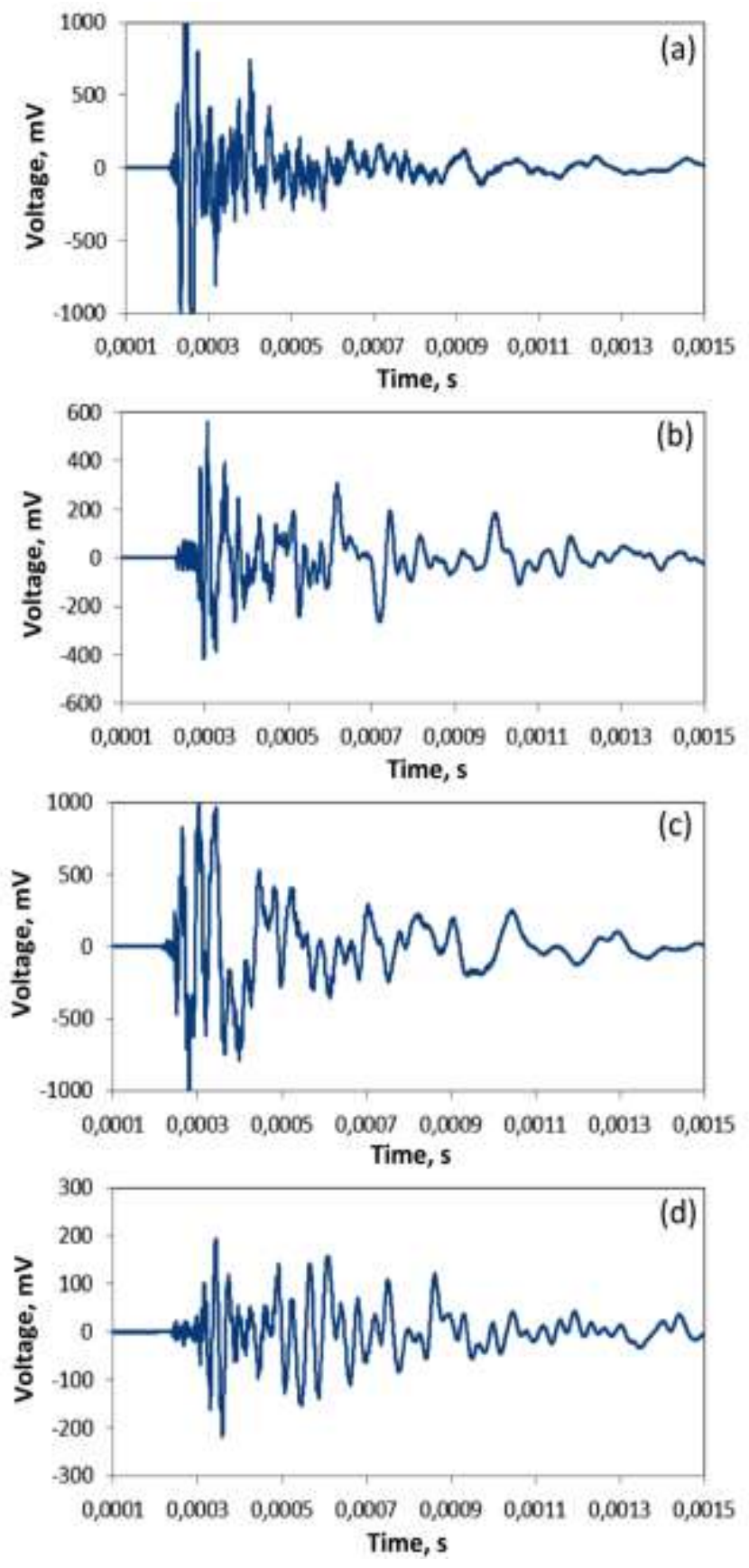

Fig. 4. AE signals registered by PZT: a) PZT 1, b) PZT 2, c) PZT 3, c) PZT 4

It is difficult to determine the event time, due to the presence of an uneven front for each signal. The most commonly used methods of the location, in which exceeding the threshold of discrimination or reaching a maximum is considered to be an occurrence of an event. These methods can be untenable by using the FOS. Group wave front does not allow one to correctly identify a maximum moment or moment of exceeding the threshold discrimination. Recently, the wavelets have been actively used to solve these problems.

For example, the spectrograms of signals using Gabor's wavelet were used to determine the phase speed in the propagation of waves in the plates $[13,14]$.
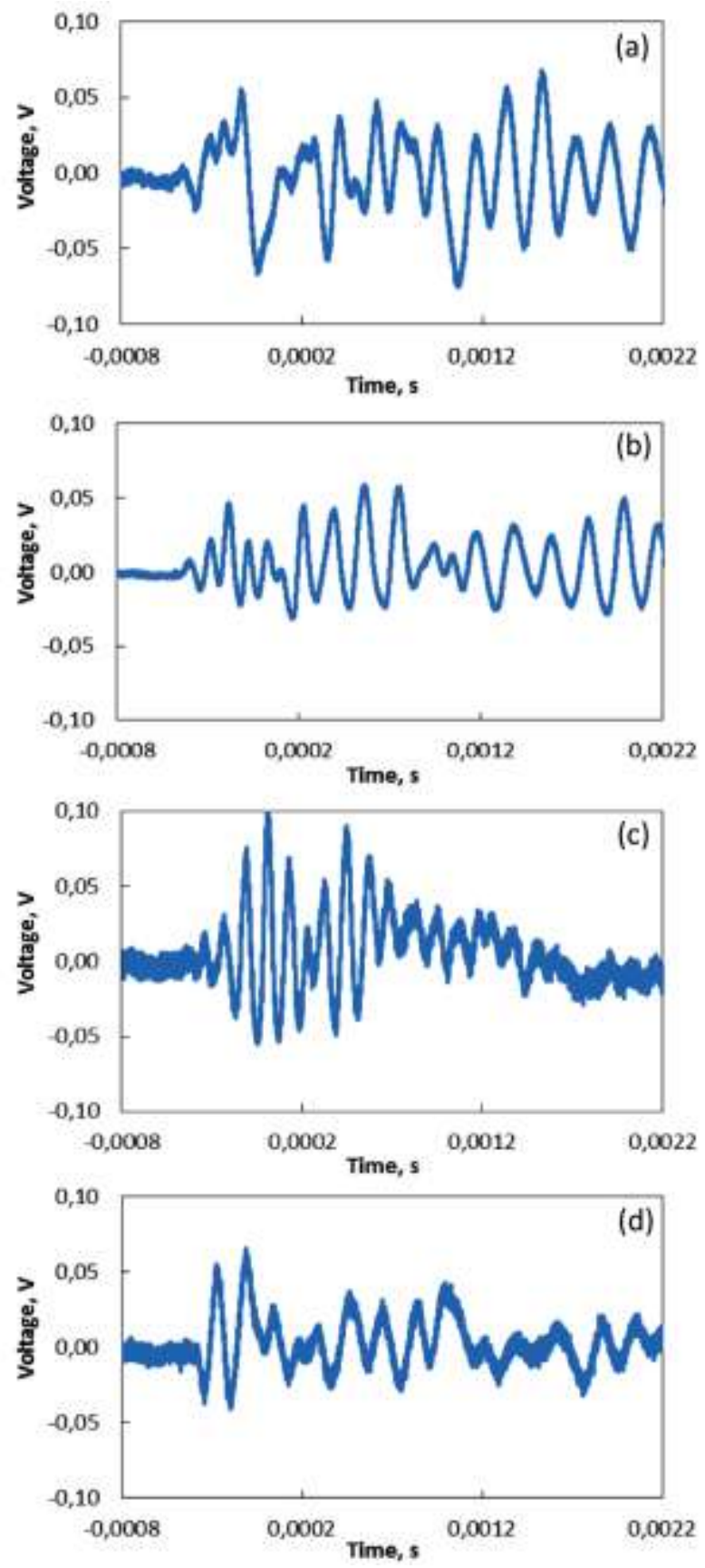

Fig. 5. AE signals registered by FOS: a) FOS 1, b) FOS 2, c) FOS 3, c) FOS 4

However, the use of a visual picture of the spectrograms is not always convenient. To determine the location of the $\mathrm{AE}$ source by one sensor, two scale components of the Daubechies wavelet decomposition were used $[15,16]$. Fig. 6 shows the wavelet spectrograms of the AE signals registered by FOS.

The Morlet wavelet was used to decompose AE signals. The signals are in the frequency range of up to $20 \mathrm{kHz}$. The main energy of the signals is concentrated in the period of up to $2000 \mu$ s. Subsequent reverberation occurs when waves are reflected from the plate edge. 

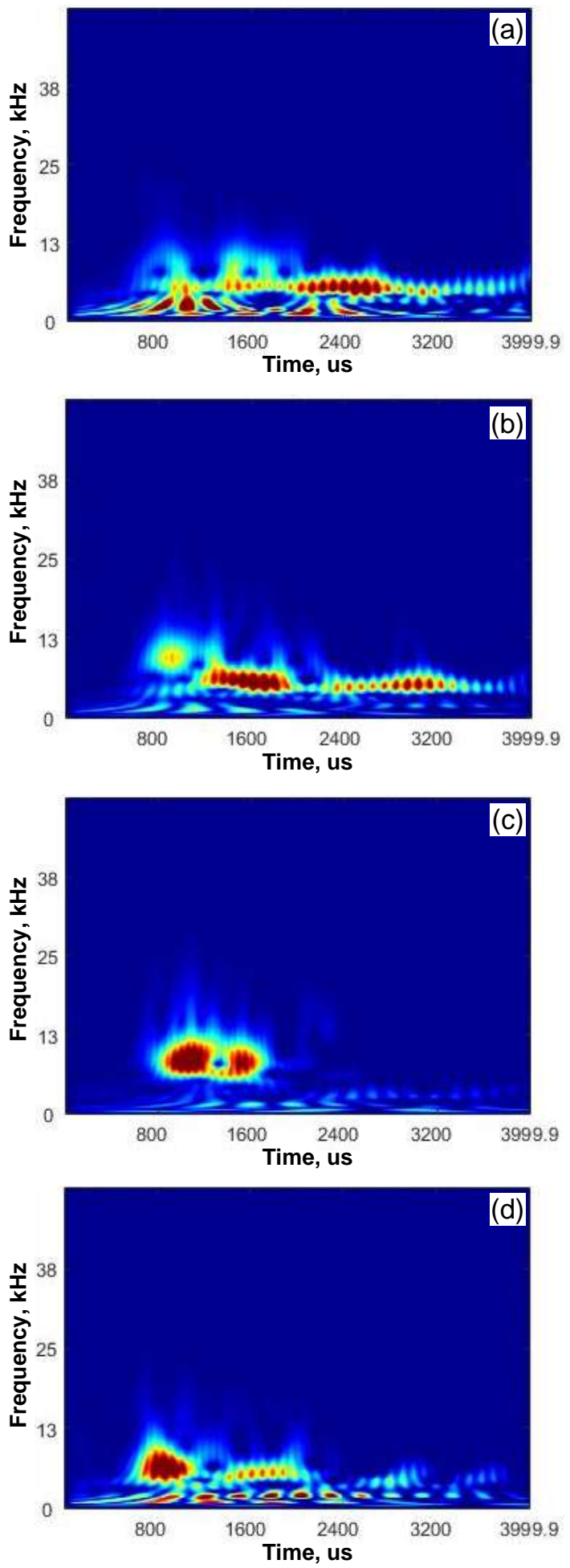

Fig. 6. Wavelet spectrograms of the AE signals registered by FOS: a) FOS 1, b) FOS 2, c) FOS 3, c) FOS 4

The spectrogram shows the reflections of the waves from the edges of PCM plate for FOS 1 and FOS 2. The main energy spectrum of the AE signals is in the range of $7-15 \mathrm{kHz}$. This is the low-frequency range for the AE signals. Numerical series of the wavelet coefficients with a maximum energy at a $13 \mathrm{kHz}$ frequency were analyzed for subsequent determination of the wave front. The graphs for the wavelet coefficients of the decomposition signals are shown in Fig. 7.
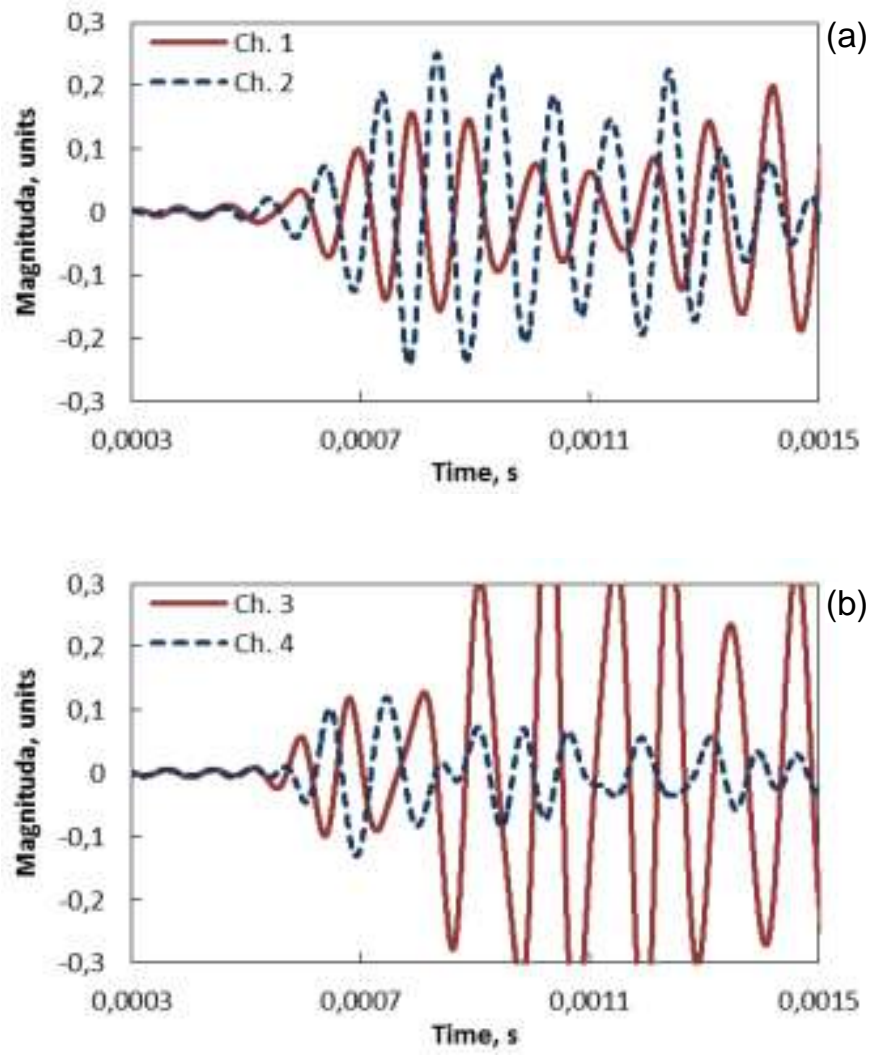

Fig. 7. Wavelet coefficients for the frequency of $13 \mathrm{kHz}$ of the $\mathrm{AE}$ signals registered by FOS: a) FOS 1, b) FOS 2, c) FOS 3, c) FOS 4

The graphs are the components of the signals allocated at the wavelet transformation by the Morlet wavelet at a frequency of $13 \mathrm{kHz}$.

The obtained graphs make it possible to estimate the wave propagation delay time. The coordinates of the FOS and the estimated time delay of signals propagation $\Delta t$ from the source to the FOS in accordance with the layout of the FOS and the signal source in Fig. 3 is shown in Table 2.

TABLE II. COORDINATES OF THE FOS AND TIME DELAY OF THE WAVE

\begin{tabular}{|l|c|c|c|c|}
\hline & FOS 1 & FOS 2 & FOS 3 & FOS 4 \\
\hline $\mathbf{X}, \mathbf{m m}$ & - & - & 220 & 0 \\
\hline $\mathbf{Y}, \mathbf{m m}$ & 0 & 75 & - & - \\
\hline $\mathbf{\Delta X}, \mathbf{m m}$ & \multicolumn{2}{|c|}{75} & \multicolumn{2}{c|}{86} \\
\hline $\mathbf{\Delta t}, \boldsymbol{\mu s}$ & \multicolumn{2}{|c|}{39} & \multicolumn{2}{c|}{1830} \\
\hline $\mathbf{c}, \mathbf{m m}$ & \multicolumn{2}{|c|}{1920} & \multicolumn{2}{c}{} \\
\hline
\end{tabular}

It follows from the calculation of the time delay of signal propagation $\Delta \mathrm{t}$ that the sound velocity along the short side (between FOS 1 and FOS 2) is $1920 \mathrm{~m} / \mathrm{s}$, and the sound velocity along the long side (between FOS 3 and FOS 4) is $1830 \mathrm{~m} / \mathrm{s}$.

The low value of the sound velocity corresponds to the antisymmetric group velocity of the Lamb waves in the PCM 
plate. In plates with the free boundaries, a family of the symmetric and antisymmetric group waves can be excited. With a small plate thickness (3 mm with 12 layers of fiberglass) only two Lamb waves (symmetric and antisymmetric zero order) can propagate. The large ultrasound attenuation in the composite material leads to the fact that the waves with large frequency values attenuate rapidly.

Due to the fact that the length of the fibers for FOS 1 and FOS 2 is greater than the length of the fibers for FOS 3 and FOS 4, the maximum duration of the integral wave signal for FOS 1 and FOS 2 is greater, therefore the maximum energy of the integral wave is recorded longer.

\section{CONCLUSION}

Thus, the possibility of registration AE signals by fiberoptic sensors based on adaptive interferometers has been established. Low frequency of the detected signals determines the features of calculating the sound velocity in the plates of polymer composite materials. Zero-order antisymmetric Lamb wave at the frequency of the maximum energy spectrum was used to determine the sound velocity.

The determination of the frequency component of the wave was carried out using the wavelet transformation by the Morlet wavelet.

\section{Acknowledgment}

This work was supported by a grant of the Russian Science Foundation (project No. 16-19-10149).

\section{References}

[1] F.G. Yuan, Structural Health Monitoring (SHM) in Aerospace Structures, Woodhead Publishing, 2016

[2] C. Boller, N. Meyendorf, "State-of-the-art in Structural Health Monitoring for Aeronautics", December 2008 [Proceedings of the International Symposium on NDT in Aerospace, 2008].

[3] A. Kesavan, S. John and I. Herszberg, "Structural Health Monitoring of Composite Structures using Artificial Intelligence Protocols", J. Intel. Mat. Sys. Str., vol. 19, is. 1, pp. 63-72, 2007.
[4] A. Kesavan, S. John and I. Herszberg, "Strain-based structural health monitoring of complex composite structures", Struct. Health Monit., vol. 7, is. 3, pp. 203-213, 2008.

[5] G. Meltz, W.W. Morey, W.H. Glenn, "Formation of Bragg gratings in optical fibers by a transverse holographic method", vol. 14, no. 15, pp. 823-825, 1989.

[6] A.A. Kamshilin, R.V. Romashko, Yu.N. Kulchin, "Adaptive interferometry with photorefractive crystals", J. Appl. Phys., vol. 105, pp. 031101, 2009

[7] S. I. Stepanov, I. A. Sokolov, "Adaptive interferometers using photorefractive crystals", pp.95-100, September 1989 [Second International Conference on Holographic Systems, Components and Applications], 1989

[8] A.A. Kamshilin, V.V. Prokofiev, "Fast adaptive interferometer with a photorefractive GaP crystal”, Opt. Lett., vol. 27, pp. 1711-1713, 2002.

[9] R.V. Romashko, Yu.N. Kulchin, A.A. Kamshilin, "A multichannel adaptive interferometry system”, J. Rus. Laser Res. vol. 31, pp. 55-60, 2010 .

[10] O.V. Bashkov, E.E. Parfenov, T.I. Bashkova, "A Soft Hardware Complex for Recording and Processing of Acoustic Signal and for Location and Identification of Their Sources", Instrum. Exp. Tech., vol. 53, pp. 682-687, 2010.

[11] O.V. Bashkov, R.V. Romashko, V.I. Zaikov, S.V. Panin, M.N. Bezruk, K. Khun, I.O. Bashkov, "Detecting Acoustic-Emission Signals with Fiber-Optic Interference Transducers", Russ. J. Nondestruct. Testing, vol. 53, no. 6, pp. 415-421, 2017.

[12] A.E. Protsenko, V.V. Telesh, "Inhibition and Cathalysis as a Method to Improve the Mechanical Properties of a Fiberglass-Reinforced Plastic Mechanics of Composite Materials”, vol. 51, pp. 555-560, 2015.

[13] M.A. Hamstad, A.O. Gallagher, J. Gary, “A wavelet transform applied to acoustic emission signals: Part 1: Source identification", J. Acoustic Emission, vol. 20, pp. 39-61, 2002.

[14] M.A. Hamstad, A.O. Gallagher, J. Gary, "A wavelet transform applied to acoustic emission signals: Part 2: Source location", J. Acoustic Emission, vol. 20, pp. 62-82, 2002.

[15] O.V. Bashkov, S.V. Panin, T.I. Bashkova, A.V. Byakov, A.A. Popkova, I.V. Shakirov, "Acoustic emission features at deformation of aluminum alloys with different strain behavior types", AIP Conf. Proc., vol. 1683 , pp. 0200231-0200234, 2015.

[16] O.V. Bashkov, R.V. Romashko, V.I. Zaykov, A.E. Protsenko, M.N. Bezruk, H. Htoo, "Detection of acoustic emission signals in the polymer composite material by adaptive fiber-optic sensors", Proc. SPIE 10176, pp. 1017613, December 2016 [Asia-Pacific Conference on Fundamental Problems of Opto- and Microelectronics, 2016]. 